\title{
Example of two different potentials which have practically the same fixed-energy phase shifts
}

\author{
Ruben G. Airapetyan ${ }^{1}$, Alexander G. Ramm *, Alexandra B. Smirnova ${ }^{2}$ \\ Department of Mathematics. Kansas State University. Manhattan, KS 66506-2602. USA
}

Received 19 May 1998; revised manuscript received 2 November 1998; accepted for publication 10 February 1999 Communicated by P.R. Holland

\begin{abstract}
The Newton-Sabatier procedure for finding the potential from fixed-energy phase shifts is analyzed. A method is proposed for finding two quite different spherically-symmetric real-valued, piecewise-constant, compactly supported potentials which generate at a fixed energy the phase shifts $\delta_{\ell}$ which are practically indistinguishable for all $\ell$. In particular, an explicit concrete example of two such potentials $q_{j}, j=1,2$, is demonstrated. These potentials have the properties: (1) sup $\left|q_{1}-q_{2}\right|>3$, and $q_{j}, j=1,2$, are of order of magnitude 1 , (2) $\delta_{l}^{(1)}=\delta_{l}^{(2)}$ for $l=0, \ldots, 4$ and $\left|\delta_{l}^{(1)}-\delta_{l}^{(2)}\right| \leqslant 10^{-5}, l>4$. (C) 1999 Elsevier Science B.V.
\end{abstract}

Keywords: Inverse scattering; Fixed energy phase shifts; Ill-posed problems; Schrödinger's equation

\section{Introduction}

Finding real-valued potentials $q(r), r=|x|$, from the set $\left\{\delta_{l}\right\}_{l=0,1, \ldots}$ of the fixed-energy phase shifts (say at $k=1$ ), is an inverse problem which was much discussed in the literature [1-3,5-7]. Physicists worked much on this problem. They used a parameter fitting procedure [5,2] which consists of the following steps:

Step 1. Given phase shifts $\delta_{l}$ at a fixed energy, which we take to be $k^{2}=1$ without loss of generality, one solves an infinite system of linear algebraic equations for some coefficients $c_{l}$ (system (12.2.7) in Ref. [2]; the references to formulas with such enumeration here and below are given always to the formulas from Ref. [2]).

Step 2. Given these coefficients $c_{l}$, one constructs some symmetric kernel ((12.2.1) in Ref. [2] and solves linear integral Eq. (12.1.2) for the kernel $K(r, \rho)$. If $K(r, \rho)$ is found, then the potential $q(r)$ is calculated by the formula (12.2.11),

\footnotetext{
* Corresponding author; e-mail: ramm@math.ksu.edu.

${ }^{1}$ E-mail: airapet@math.ksu.edu.

2E-mail: smirn@math.ksu.edu.
} 


$$
q(r)=-\frac{2}{r} \frac{\mathrm{d}}{\mathrm{d} r} \frac{K(r, r)}{r}
$$

This procedure is presented in Refs. [5,2] as an inversion method and many physicists consider it as one. In this introduction we analyze this procedure.

Let us define an inversion method as a method which satisfies the following conditions:

(1) given the exact data, corresponding to a $q(r) \in Q$, where $Q$ is a class of potentials in which the inverse scattering problem is uniquely solvable, one can carry through theoretically (and not necessarily computationally) each step of the method (we neglect here the numerical errors unavoidable in the numerical implementation of any method),

(2) the method yields the unique solution $q \in Q$ to the inverse problem.

In this paper we take the class $Q$ of compactly supported real-valued potentials $q(r)$ which satisfy the conditions: $\int_{0}^{a}|q(r)|^{2} r^{2} \mathrm{~d} r<\infty, q(r)=0$ for $r>a$, where $a>0$ is an arbitrary large fixed number which is called the range of the potential. For $q \in Q$ the inverse scattering problem with fixed-energy phase shifts as the scattering data has a unique solution [7]. Let us assume that the given scattering data come from a potential in this class. The question is: can one reconstruct such a potential using the Newton-Sabatier (NS) procedure and assume that this procedure can be carried through? We prove that the answer to the above question is no.

Solving the inverse scattering problem includes the requirement that the potential obtained by an inversion method produces the original data from which it was reconstructed. In Ref. [2] this property is called consistency of the method.

The basic results of our paper are:

(1) It is shown that if one applies the NS procedure to the data generated by a potential $q \in Q$, then, assuming that this procedure can be carried through, it yields the potentials $q(r)$ which are analytic in a neighborhood of the positive semi-axis of the complex $r$-plane and may have a simple pole at the origin, and therefore is not equal to the potential $q \in Q$ with which we started.

(2) It is shown that the stability estimate (see estimate (1.5) below) from Ref. [8] is practically accurate: an example is constructed of two different piecewise-constant compactly supported potentials which produce practically the same (up to the error of order $10^{-5}$ ) fixed-energy phase shifts for all $\ell$.

Therefore even if both steps in the NS method with exact data, corresponding to a $q \in Q$, can be carried through in the above class $Q$, they lead to a potential $q_{1}(r) \notin Q$, which has a very special property (P), namely, the function $r q(r)$ has to be analytic in a neighborhood of the positive semiaxis of the complex $r$-plane. This property the potentials from class $Q$ do not have.

Consistency of the NS procedure is not proved in Refs. [2,5]: assuming that the NS procedure can be carried through for the given data, there is no guarantee that the potential generated by this procedure produces the same data with which one started.

In Ref. [2, p. 205] an argument is given to prove the consistency of the NS procedure, but this argument is not completely convincing because: (1) it is not quite clear why $A_{\ell}^{\prime}$ and $\delta_{\ell}^{\prime}$ solve Eq. (12.2.5), (2) existence and uniqueness of the solution to Goursat-type problem (11.2.5)-(11.2.7) are not proved in Ref. [2]; since the coefficients in this problem are variable and have non-integrable singularity at the origin, the desired uniqueness and existence do not follow from the known results; in the literature one can find results on the existence and uniqueness of the transformation kernels for Eq. (11.1.2), but these kernels depend on $\ell$, while the transformation kernel used in Refs. [2,5] should be independent on $\ell$.

Note that the basic ansatz of the NS procedure says that the transformation kernel (12.2.3) is generated by the symmetric kernel (12.2.1) and condition (12.2.2) holds.

There is no guarantee that this ansatz can yield (by solving system (12.2.4) and then using formula (12.2.11)) a potential which can produce generic scattering data. Indeed, as we prove below, the potential which is obtained by the NS procedure (in the case when this procedure can be carried through) has to satisfy property $(P)$, which generic potentials do not have. Therefore the usage of this ansatz is not fully justified: there is no 
guarantee that one can fit the exact phase shifts, generated by a potential $q \in Q$, using the potentials produced by the NS procedure based on the above ansatz.

In the discussion of these principal points we are not talking at all about the difficulties which come from the fact that in practice the data are never exact.

There is no guarantee that the steps 1 and 2 of the NS procedure with exact data can he carried through, in general, because:

(1) System (12.2.7) may have, in general, no solution in the class (12.2.2),

(2) Integral Eq. (12.1.2) for the kernel $K(r, \rho)$ may be not solvable for some (not too small) $r>0$.

Note that existence of the transformation kernel (11.2.4) is not proved in Refs. [2,5] but was proved recently in Ref. [11].

It is easy to prove that Eq. (12.1.2) is uniquely solvable for sufficiently small $r>0$ since in this case the norm of the corresponding integral operator in $C(0, r)$ tends to 0 as $r \rightarrow 0$.

If it is possible to solve (12.2.7) in the class (12.2.2) and Eq. (12.1.2) is uniquely solvable for all $r>0$, then the potential $(12.2 .11)$ must have the following special property:

(P) The function $r q(r)$ must be an analytic function of $r$ in a neighborhood of the positive semiaxis $(0, \infty)$.

The proof of property $(\mathrm{P})$ for the potentials obtained by the NS scheme is given below.

From the logical point of view property $(\mathrm{P})$ is sufficient for the proof of our hasic conclusion:

Conclusion: For the generic data corresponding to a compactly supported potential $q(r) \in Q Q$ the NS procedure cannot reproduce the original potential.

Thus, we have the following logical possibilities:

(i) Either the NS procedure can be carried through for the phase shifts corresponding to a $q \in Q$ and the reconstructed by this procedure potential $q_{1}(r)$ reproduces the original phase shifts, and then the inverse scattering problem has more than one solution and the solution $q \in Q$ cannot be obtained by the NS procedure;

(ii) or the NS procedure cannot be carried through for the phase shifts corresponding to $q \in Q$ :

(iii) or the NS procedure can be carried through for these phase shifts but the obtained potential $q_{1}(r)$ does not generate the original phase shifts, in which case the NS procedure does not solve the inverse scattering problem.

Similar argument holds for any class of potentials which do not have property $(P)$ and for which the uniqueness of the solution to the inverse scattering problem with exact data is valid.

Let us now prove that the potential, obtained by the NS procedure, assuming that this procedure can be carried through for the given data, must have property $(\mathrm{P})$.

Proof of property $(P)$. The functions $u_{\ell}$ in (12.2.3) are entire functions of $r$ which have the following known asymptotics for large $\ell: u_{\ell} \sim \sqrt{(r / 2)}(e r /(2 \ell+1))^{(2 \ell+1) / 2}(2 \ell+1)^{-1 / 2}$. Therefore the function $(12.2 .1)$, with $c_{f}$ satisfying (12.2.2), is an entire function of $r$ and $r^{\prime}$.

Let us first prove that $r q(r)$ is analytic in a neighborhood of the origin, since this claim already is sufficient for a justification of our basic conclusion and the proof of this claim is quite easy.

The system (12.2.4) has the matrix $L_{\ell \ell^{\prime}}(r)=\int_{0}^{r} u_{\ell}(\rho) u_{\ell^{\prime}}(\rho) \rho^{-2} \mathrm{~d} \rho$ (see formula (11.4.5) with $V_{0}=0$ ). Since $u_{f}(r)$ is an entire function of $r$ and is $O\left(r^{\ell+1}\right)$ as $r \rightarrow 0$, one concludes that $L_{\ell \ell^{\prime}}(r)$ is an entire function of $r$. For sufficiently small $r$ system (12.2.4) has dominating diagonal terms and therefore is uniquely solvable by iterations. Since $u_{\ell}(r)$ and $L_{\ell \ell^{\prime}}(r)$ are entire functions of $r$, the Neumann series yields the solution $\varphi_{\ell}(r)$ of (12.2.4) which is an analytic function of $r$ in a neighborhood of the point $r=0$. This implies that the kernel $K\left(r, r^{\prime}\right)$, defined by (12.2.3), is analytic with respect to both variables in a neighborhood of $r=0, r^{\prime}=0$. Therefore $K(r, r)$ is analytic in a neighborhood of $r=0$ and consequently the potential (12.2.11) (defined by formula (1.1)) is analytic in a punctured at $r=0$ neighborhood of the origin and the function $r q(r)$ is analytic 
in a neighborhood of the origin. Our first claim is proved.

Let us now prove that $r q(r)$ is analytic in a neighborhood of the positive semiaxis provided that Eq. (12.1.2) is uniquely solvable. The kernel and the free term of this equation, as we have proved above, are entire functions of $r$ and $r^{\prime}$. It is known that the solution of a Fredholm second kind integral equation whose kernel and free term depend on a parameter $z$ analytically in a certain region $\Delta$ of a complex plane, is analytic in a neighborhood of a point $z_{0} \in \Delta$, provided that the above Fredholm equation is uniquely solvable at $z=z_{0}$ (see Ref. [10] for a more general result).

Let us introduce the new variables $\rho=r s, r^{\prime}=r t$ in Eq. (12.1.2) with the aim to get an equation with a fixed integration region. Eq. (12.1.2) becomes

$$
K(r, r t)=f(r, r t)-\int_{0}^{1} K(r, r s) f(r s, r t) r^{-1} s^{-2} \mathrm{~d} s .
$$

Let

$$
a(s, t, r):=f(r s, r t)(r s t)^{-1}, \quad K(r, r s) s^{-1}:=b(s, r), \quad f(r, r t) t^{-1}:=g(r, t) .
$$

Then the above equation takes the form

$$
b(t, r)=g(r, t)-\int_{0}^{1} a(s, t, r) b(s, r) \mathrm{d} s .
$$

This equation is equivalent to Eq. (12.1.2) in Ref. [2]. It is a Fredholm second kind equation whose kernel $a(s, t, r)$ and the free term $g(r, t)$ depend on the parameter $r$ analytically on the whole complex $r$-plane because the kernel $f(s, t)$ is an entire function of $s$ and $t$, as we proved above, and it vanishes at $s=0$ and at $t=0$. Also $g(r, t)$ and $a(s, t, r)$ are entire functions of $t$. If the NS procedure can be carried through, then this equation has to be uniquely solvable for any $r>0$. Therefore its solution is an analytic function of $r$ in a neighborhood of positive semiaxis $(0, \infty)$ and an analytic function of $t$ on the whole complex $t$-plane. This implies that the kernel $K(r, r)$ is an analytic function of $r$ in a neighborhood of the positive semiaxis $(0, \infty)$. Thus, the potential $(12.2 .11)$ has property $(P)$. The proof is complete.

Therefore, in general, the inverse scattering problem is not solvable in the class $Q$ by means of the NS procedure.

The uniqueness of the recovery of $q(r)$ from the fixed-energy phase shifts $\delta_{l}, l \geqslant 0$, is not established in Ref. [2]. Such a uniqueness theorem follows for $q \in Q$ from the uniqueness theorem proved in Ref. [7] for potentials which are not necessarily spherically symmetric.

No such theorem is known for potentials which decay as some power of $1 / r$, that is,

$$
|q|=O\left(\frac{1}{(1+r)^{m}}\right), \quad m>2 \text { is fixed. }
$$

It is known (see Ref. [9]) that the range $a$ of a compactly supported potential, which does not change sign in some neighborhood $(a-\varepsilon, a)$, can be calculated in terms of $\left\{\delta_{l}\right\}$,

$$
a=\lim _{l \rightarrow \infty}\left(\frac{2 l+1}{e}\left|\delta_{l}\right|^{1 / 2 l}\right) .
$$

It follows from (1.3) that 


$$
\left|\delta_{l}\right| \leqslant c\left(\frac{a e[1+o(1)]}{2 l+1}\right)^{2 l}, \quad c=\mathrm{const}>0 .
$$

This implies that $\delta_{l}$ decay very fast as $l \rightarrow \infty$, provided that $q(r)$ is compactly supported.

If $q(r)$ decays on a power scale (1.2) then $\delta_{l}$ decays also as some positive power of $l^{-1}$ (see Ref. [4]).

In this paper we construct two piecewise-constant, real-valued, compactly supported potentials $q_{1}(r)$ and $q_{2}(r)$, which are quite different (see Table 1 in Section 3 ) and which generate practically identical phase shifts $\delta_{1}$.

The potentials $q_{j}, j=1,2$, are constructed by a computer code, which recovers $q(r)$ (in the above class of potentials) from the data $\left\{\delta_{l}\right\}, l \geqslant 0$.

It is known that the inverse problem of quantum scattering with fixed-energy scattering data is very ill-posed. if the energy is not very high, and the numerical reconstruction of the potential from noisy scattering data is quite difficult.

However, our numerical results also show that if an initial approximation to the unknown potential is known with relatively small error $\left(\approx 10^{-1}\right)$, then the recovery of $q(r)$ is possible with high accuracy $\left(10^{-14}\right)$.

In Ref. [8] a mathematically justified method is proposed for stable inversion of fixed-energy scattering data for not necessarily spherically symmetric potentials with compact support. If the potential $q(r)$ is hounded spherically, symmetric and has compact support then the result in Ref. [8] yields an algorithm for reconstruction of $q(r)$ from the phase shifts $\delta_{l}$ known with some error $\varepsilon$. This algorithm yields $\hat{q}_{\varepsilon}$ such that

$$
\sup _{\xi}\left|\tilde{q}(\xi)-\hat{q}_{\varepsilon}\right| \leqslant c \frac{(\ln |\ln \varepsilon|)^{2}}{|\ln \varepsilon|}, \quad \varepsilon \rightarrow 0 .
$$

where $c>0$ is some constant which does not depend on $\varepsilon$, but does depend on some norm of the potcntial, $\tilde{q}(\xi)$ is the Fourier transform of $q(x)$. The right-hand side of (1.5) tends to zero as $\varepsilon \rightarrow 0$, however it does so very slowly. Therefore practically one can hope to recover $q(r)$ from the fixed-energy scattering data, that is, from the phase shifts $\delta_{l}$ at a fixed $k$, only if either the class of the potentials is a priori chosen to be rather narrow, for instance, finite-parametric, or the accuracy of the data is very high. Our numerical results confirm these conclusions. The example we constructed is an illustration of the stability estimate (1.5).

In Section 2 a numerical method is described which we use to invert the fixed-energy phase shifts for the potential $q(r)$. In Section 3 the numerical results are presented.

\section{Numerical method}

Consider a finite set of points $0=r_{0}<r_{1}<r_{2}<\ldots<r_{N}=R$ and a piecewise-constant potential

$$
q(r)=q_{i}, \quad \text { on }\left[r_{i-1}, r_{i}\right) \text { for } i=1, \ldots, N, \quad \text { and } q=0, \quad \text { for } r \geqslant R \text {. }
$$

Denote $\kappa_{i}^{2}:=k^{2}-q_{i}$, where $i=1, \ldots, N$, and $k$ is some fixed positive number. Consider the following problem for the radial Schrödinger equation,

$$
\frac{\mathrm{d}^{2} \varphi_{l}}{\mathrm{~d} r^{2}}+\left(k^{2}-\frac{l(l+1)}{r^{2}}\right) \varphi_{l}=q \varphi_{l}, \quad \lim _{r \rightarrow 0}(2 l+1) ! ! r^{-l-1} \varphi_{l}(r)=1,
$$

which we rewrite as

$$
\frac{\mathrm{d}^{2} \varphi_{I}}{\mathrm{~d} r^{2}}+\left(\kappa_{i}^{2}-\frac{l(l+1)}{r^{2}}\right) \varphi_{l}=0
$$


on the interval $r_{i-1} \leqslant r<r_{i}$. On $\left[r_{i-1}, r_{i}\right)$ one has the following general solution of (2.3),

$$
\varphi_{l}(r)=A_{i} u_{l}\left(\kappa_{i} r\right)+B_{i} v_{l}\left(\kappa_{i} r\right),
$$

where

$$
u_{l}(z)=\sqrt{\frac{\pi z}{2}} J_{l+1 / 2}(z), \quad v_{l}(z)=\sqrt{\frac{\pi z}{2}} Y_{l+1 / 2}(z) .
$$

We assume below that $\kappa_{i}$ does not vanish for all $i$. If $\kappa_{i}=0$ for some $i$ then our approach is still valid with obvious changes.

From the regularity of $\varphi_{l}$ at zero one gets $B_{1}=0$. Denote $x_{i}=B_{i} / A_{i}$, then $x_{1}=0$. We are looking for the continuously differentiable solution $\varphi_{l}$. Thus, the following interface conditions hold,

$$
\begin{aligned}
& A_{i} u_{l}\left(\kappa_{i} r_{i}\right)+B_{i} v_{l}\left(\kappa_{i} r_{i}\right)=A_{i+1} u_{l}\left(\kappa_{i+1} r_{i}\right)+B_{i+1} v_{l}\left(\kappa_{i+1} r_{i}\right), \\
& \frac{\kappa_{i}}{\kappa_{i+1}}\left[A_{i} u_{l}^{\prime}\left(\kappa_{i} r_{i}\right)+B_{i} v_{l}^{\prime}\left(\kappa_{i} r_{i}\right)\right]=A_{i+1} u_{l}^{\prime}\left(\kappa_{i+1} r_{i}\right)+B_{i+1} v_{l}^{\prime}\left(\kappa_{i+1} r_{i}\right) .
\end{aligned}
$$

The Wronskian $W\left(u_{l}(r), v_{l}(r)\right)=1$, thus

$$
\begin{aligned}
& A_{i+1}=v_{l}^{\prime}\left(\kappa_{i+1} r_{i}\right)\left[A_{i} u_{l}\left(\kappa_{i} r_{i}\right)+B_{i} v_{l}\left(\kappa_{i} r_{i}\right)\right]-\frac{\kappa_{i}}{\kappa_{i+1}} v_{l}\left(\kappa_{i+1} r_{i}\right)\left[A_{i} u_{l}^{\prime}\left(\kappa_{i} r_{i}\right)+B_{i} v_{l}^{\prime}\left(\kappa_{i} r_{i}\right)\right], \\
& B_{i+1}=\frac{\kappa_{i}}{\kappa_{i+1}} u_{l}\left(\kappa_{i+1} r_{i}\right)\left[A_{i} u_{l}^{\prime}\left(\kappa_{i} r_{i}\right)+B_{i} v_{l}^{\prime}\left(\kappa_{i} r_{i}\right)\right]-u_{l}^{\prime}\left(\kappa_{i+1} r_{i}\right)\left[A_{i} u_{l}\left(\kappa_{i} r_{i}\right)+B_{i} v_{l}\left(\kappa_{i} r_{i}\right)\right] .
\end{aligned}
$$

Therefore

$$
\left(\begin{array}{l}
A_{i+1} \\
B_{i+1}
\end{array}\right)=\frac{1}{\kappa_{i+1}}\left(\begin{array}{cc}
\alpha_{11}^{i} & \alpha_{12}^{i} \\
\alpha_{21}^{i} & \alpha_{22}^{i}
\end{array}\right)\left(\begin{array}{c}
A_{i} \\
B_{i}
\end{array}\right),
$$

where the entries of the matrix $\alpha^{\prime}$ can be written explicitly,

$$
\begin{aligned}
& \alpha_{11}^{i}=\kappa_{i+1} u_{l}\left(\kappa_{i} r_{i}\right) v_{l}^{\prime}\left(\kappa_{i+1} r_{i}\right)-\kappa_{i} u_{l}^{\prime}\left(\kappa_{i} r_{i}\right) v_{l}\left(\kappa_{i+1} r_{i}\right), \\
& \alpha_{12}^{i}=\kappa_{i+1} v_{l}\left(\kappa_{i} r_{i}\right) v_{l}^{\prime}\left(\kappa_{i+1} r_{i}\right)-\kappa_{i} v_{l}^{\prime}\left(\kappa_{i} r_{i}\right) v_{l}\left(\kappa_{i+1} r_{i}\right), \\
& \alpha_{21}^{i}=\kappa_{i} u_{l}^{\prime}\left(\kappa_{i} r_{i}\right) u_{l}\left(\kappa_{i+1} r_{i}\right)-\kappa_{i+1} u_{l}\left(\kappa_{i} r_{i}\right) u_{l}^{\prime}\left(\kappa_{i+1} r_{i}\right), \\
& \alpha_{22}^{i}=\kappa_{i} v_{l}^{\prime}\left(\kappa_{i} r_{i}\right) u_{l}\left(\kappa_{i+1} r_{i}\right)-\kappa_{i+1} v_{l}\left(\kappa_{i} r_{i}\right) u_{l}^{\prime}\left(\kappa_{i+1} r_{i}\right) .
\end{aligned}
$$

Thus

$$
x_{i+1}=\frac{\alpha_{21}^{i}+\alpha_{22}^{i} x_{i}}{\alpha_{11}^{i}+\alpha_{12}^{i} x_{i}}, \quad x_{i}:=\frac{B_{i}}{A_{i}}
$$

Denote the phase shift $\delta(l, k)$ by the formula

$$
\varphi_{l}(r) \sim \frac{|F(l, k)|}{k^{l+1}} \sin \left(k r-\frac{\pi l}{2}+\delta(l, k)\right), \quad r \rightarrow \infty,
$$

where $F(l, k)$ is the Jost function. For $r>R$

$$
\varphi_{l}(r)=A_{N+1} u_{l}(k r)+B_{N+1} v_{l}(k r) .
$$


From (2.11) and the asymptotics $u_{l}(k r) \sim \sin (k r-l \pi / 2), v_{l}(k r) \sim-\cos (k r-l \pi / 2), r \rightarrow \infty$, one gets

$$
\tan \delta(k, l)=-\frac{B_{N+1}}{A_{N+1}}=-x_{N+1}, \quad \delta(k, l)=-\arctan x_{N+1} .
$$

Now let us assume that the column vector $\delta^{*}=\left(\delta_{0}^{*}, \ldots, \delta_{N-1}^{*}\right)$ is given and introduce the nonlincar operator $\Phi: R^{N} \rightarrow R^{N}$, which maps a column vector $\kappa=\left(\kappa_{1}, \ldots, \kappa_{N}\right)$ into a vector $\Phi(\boldsymbol{\kappa})=\delta-\delta^{*}$, where $\delta$ is given by (2.12) and $\delta_{j}^{*}, j=0, \ldots, N-1$, are given fixed energy phase shifts. Thus, we are looking for the solution to the problem

$$
\Phi(\boldsymbol{\kappa})=0 .
$$

In order to solve this equation by means of the Newton method, first one has to find the Jacobian $\left\|\Phi^{\prime} \mid=\right\| \partial_{j} \Phi_{i} \|$. where $\partial_{j}=\partial / \partial \kappa_{j}$.

Since $\alpha^{i}=\alpha^{i}\left(\kappa_{i}, \kappa_{i+1}\right), x_{i}=x_{i}\left(\kappa_{1}, \ldots, \kappa_{i}\right)$, one has

$$
\partial_{j} x_{i+1}=\frac{\operatorname{det} \alpha^{i}}{\left(\alpha_{11}^{i}+\alpha_{12}^{i} x_{i}\right)^{2}} \partial_{j} x_{i}, \quad j<i,
$$

and, for $j=i$ or $j=i+1$, one gets

$$
\begin{aligned}
& \partial_{j} x_{i+1}=\frac{\operatorname{det} \alpha^{i}}{\left(\alpha_{11}^{i}+\alpha_{12}^{i} x_{i}\right)^{2}} \partial_{j} x_{i} \\
& +\frac{\left(\alpha_{11}^{i}+\alpha_{12}^{i} x_{i}\right)\left(\partial_{j} \alpha_{21}^{i}+x_{i} \partial_{j} \alpha_{22}^{i}\right)-\left(\alpha_{21}^{i}+\alpha_{22}^{i} x_{i}\right)\left(\partial_{j} \alpha_{11}^{i}+x_{i} \partial_{j} \alpha_{12}^{i}\right)}{\left(\alpha_{11}^{i}+\alpha_{12}^{i} x_{i}\right)^{2}},
\end{aligned}
$$

where

$$
\begin{aligned}
& \partial_{i} \alpha_{11}^{i}=\kappa_{i+1} r_{i} u_{l}^{\prime}\left(\kappa_{i} r_{i}\right) v_{l}^{\prime}\left(\kappa_{i+1} r_{i}\right)-u_{l}^{\prime}\left(\kappa_{i} r_{i}\right) v_{l}\left(\kappa_{i+1} r_{i}\right)-\kappa_{i} r_{i} u_{l}^{\prime \prime}\left(\kappa_{i} r_{i}\right) v_{l}\left(\kappa_{i+1} r_{i}\right), \\
& \partial_{i} \alpha_{12}^{\prime}=\kappa_{i+1} r_{i} v_{l}^{\prime}\left(\kappa_{i} r_{i}\right) v_{l}^{\prime}\left(\kappa_{i+1} r_{i}\right)-v_{l}^{\prime}\left(\kappa_{i} r_{i}\right) v_{l}\left(\kappa_{i+1} r_{i}\right)-\kappa_{i} r_{i} v_{l}^{\prime \prime}\left(\kappa_{i} r_{i}\right) v_{l}\left(\kappa_{i+1} r_{i}\right), \\
& \partial_{i} \alpha_{21}^{\prime}=u_{l}^{\prime}\left(\kappa_{i} r_{i}\right) u_{l}\left(\kappa_{i+1} r_{i}\right)+\kappa_{i} r_{i} u_{l}^{\prime \prime}\left(\kappa_{i} r_{i}\right) u_{l}\left(\kappa_{i+1} r_{i}\right)-\kappa_{i+1} r_{i} u_{l}^{\prime}\left(\kappa_{i} r_{i}\right) u_{l}^{\prime}\left(\kappa_{i+1} r_{i}\right), \\
& \partial_{i} \alpha_{22}^{i}=v_{l}^{\prime}\left(\kappa_{i} r_{i}\right) u_{l}\left(\kappa_{i+1} r_{i}\right)+\kappa_{i} r_{i} v_{l}^{\prime \prime}\left(\kappa_{i} r_{i}\right) u_{l}\left(\kappa_{i+1} r_{i}\right)-\kappa_{i+1} r_{i} v_{l}^{\prime}\left(\kappa_{i} r_{i}\right) u_{l}^{\prime}\left(\kappa_{i+1} r_{i}\right), \\
& \partial_{i+1} \alpha_{11}^{i}=u_{l}\left(\kappa_{i} r_{i}\right) v_{l}^{\prime}\left(\kappa_{i+1} r_{i}\right)+\kappa_{i+1} r_{i} u_{l}\left(\kappa_{i} r_{i}\right) v_{l}^{\prime \prime}\left(\kappa_{i+1} r_{i}\right)-\kappa_{i} r_{i} u_{l}^{\prime}\left(\kappa_{i} r_{i}\right) v_{l}^{\prime}\left(\kappa_{i+1} r_{i}\right), \\
& \lambda_{i+1} \alpha_{12}^{i}=v_{l}\left(\kappa_{i} r_{i}\right) v_{l}^{\prime}\left(\kappa_{i+1} r_{i}\right)+\kappa_{i+1} r_{i} v_{l}\left(\kappa_{i} r_{i}\right) v_{l}^{\prime \prime}\left(\kappa_{i+1} r_{i}\right)-\kappa_{i} r_{i} v_{l}^{\prime}\left(\kappa_{i} r_{i}\right) v_{l}^{\prime}\left(\kappa_{i+1} r_{i}\right), \\
& \partial_{i+1} \alpha_{21}^{\prime}=\kappa_{i} r_{i} u_{l}^{\prime}\left(\kappa_{i} r_{i}\right) u_{l}^{\prime}\left(\kappa_{i+1} r_{i}\right)-u_{l}\left(\kappa_{i} r_{i}\right) u_{l}^{\prime}\left(\kappa_{i+1} r_{i}\right)-\kappa_{i+1} r_{i} u_{l}\left(\kappa_{i} r_{i}\right) u_{l}^{\prime \prime}\left(\kappa_{i+1} r_{i}\right), \\
& \partial_{i+1} \alpha_{22}^{i}=\kappa_{i} r_{i} v_{l}^{\prime}\left(\kappa_{i} r_{i}\right) u_{l}^{\prime}\left(\kappa_{i+1} r_{i}\right)-v_{l}\left(\kappa_{i} r_{i}\right) u_{l}^{\prime}\left(\kappa_{i+1} r_{i}\right)-\kappa_{i+1} r_{i} v_{l}\left(\kappa_{i} r_{i}\right) u_{l}^{\prime \prime}\left(\kappa_{i+1} r_{i}\right),
\end{aligned}
$$

Then one solves problem (2.13) iteratively,

$$
\boldsymbol{\kappa}^{j+1}=\boldsymbol{\kappa}^{j}-\gamma\left[\Phi^{\prime}\left(\boldsymbol{\kappa}^{j}\right)\right]^{-1} \Phi\left(\boldsymbol{\kappa}^{j}\right), \quad j=0,1, \ldots, \quad \boldsymbol{\kappa}^{j}:=\left(\boldsymbol{\kappa}_{1}^{j}, \ldots, \boldsymbol{\kappa}_{N}^{j}\right),
$$

where the step $\gamma$ is chosen small enough. One stops the iterative process when $\left\{\Phi\left(\boldsymbol{\kappa}^{j}\right) \|\right.$ becomes smaller than some small threshold $\epsilon$.

Instead of inverting matrix $\Phi^{\prime}$ in (2.16) one denotes $h^{j}:=\left[\Phi^{\prime}\left(\boldsymbol{\kappa}^{j}\right)\right]^{-1} \Phi\left(\boldsymbol{\kappa}^{j}\right)$ and uses the Gauss process with the choice of the maximal element by lines and columns of the corresponding linear system,

$$
\left[\Phi^{\prime}\left(\kappa^{j}\right)\right]_{l, m} \boldsymbol{h}_{m}^{j}=\Phi_{l}\left(\boldsymbol{\kappa}^{j}\right), \quad \boldsymbol{\kappa}^{j+1}=\kappa^{j}-\gamma \boldsymbol{h}^{j} .
$$

If at a step $l_{0}$ of the Gauss process, the maximal element of the remaining elements of the matrix becomes smaller than some threshold $\epsilon_{1}$ one sets all the remaining quantities $\boldsymbol{h}_{l}^{u}, l>l_{0}$ equal to zero. 
Table 1

\begin{tabular}{lllrr}
\hline$r_{i}$ & $q_{\text {orig }}$ & $\delta_{l}$ & $\tilde{\delta}_{l}$ & $q_{\text {rec }}$ \\
\hline 0.5 & 0.6907240 & $-9.941752 \times 10^{-2}$ & $-9.941752 \times 10^{-2}$ & -2.415259 \\
1 & 0.2084572 & $-3.779873 \times 10^{-2}$ & $-3.779873 \times 10^{-2}$ & 1.558406 \\
1.5 & $5.330886 \times 10^{-2}$ & $-1.179639 \times 10^{-2}$ & $-1.179639 \times 10^{-2}$ & -0.589802 \\
2 & $1.069364 \times 10^{-2}$ & $-3.014222 \times 10^{-3}$ & $-3.014222 \times 10^{-3}$ & 0.355841 \\
2.5 & $1.339883 \times 10^{-3}$ & $-6.494566 \times 10^{-4}$ & $-6.494566 \times 10^{-4}$ & -0.171777 \\
3 & $2.480612 \times 10^{-5}$ & $-1.691416 \times 10^{-4}$ & $-1.719357 \times 10^{-4}$ & $8.157301 \times 10^{-2}$ \\
3.5 & $5.794400 \times 10^{-5}$ & $-1.026825 \times 10^{-4}$ & $-9.611266 \times 10^{-5}$ & $-3.368591 \times 10^{-2}$ \\
4 & $1.161896 \times 10^{-4}$ & $-7.547580 \times 10^{-5}$ & $-6.558222 \times 10^{-5}$ & $1.191644 \times 10^{-2}$ \\
4.5 & $9.739553 \times 10^{-5}$ & $-4.157540 \times 10^{-5}$ & $-3.745421 \times 10^{-5}$ & $-3.106548 \times 10^{-3}$ \\
5 & $5.827817 \times 10^{-5}$ & $-1.675351 \times 10^{-5}$ & $-2.219373 \times 10^{-5}$ & $5.785574 \times 10^{-4}$ \\
\hline
\end{tabular}

\section{Numerical results}

As was pointed out in the introduction, the principal difficulty of the numerical realization of the inversion schemes, developed for the fixed cncrgy inverse scattering problem, is the fast decay of the phase shifts $\delta_{l}$. Therefore one cannot discriminate between the noise and the data starting from relatively small $l$. So one can use for the numerical reconstruction of a potential very few phase shifts $\delta_{0}, \ldots, \delta_{t_{0}}$, thus neglecting the rest, which we call the "tail" $\delta_{l}, l>l_{0}$. The goal of our numerical experiments is to show numerically that in general the "tail" of the data can not be neglected.

In the numerical experiments we start with some piecewise-constant potential (2.1) and take $N=10$. For example let us start with the following potential,

$$
q_{i}=(1+\cos (i / 2)) \mathrm{e}^{-\infty}, \quad i=1,2, \ldots, 10 .
$$

This potential is denoted in Table 1 by $q_{\text {orig. }}$. Then, using formulas (2.9), (2.12) we calculate the phase shifts $\delta_{l}$, $l=0,1, \ldots, 9$, corresponding to some fixed value of $k(k=2)$. Then taking the first five phase shifts $\delta_{0}, \ldots, \delta_{4}$ unchanged, we perturb the phase shifts $\delta_{5}, \ldots, \delta_{9}$ a little, by a random quantity of order of magnitude $\sim 10^{-5}$. This corresponds to the order of $\delta_{5}$. By the numerical method described in the previous section (with $\epsilon_{1}=10^{-10}$ and $\gamma=1$ ) we reconstruct the piecewise-constant potential (denoted in Table 1 as $q_{\mathrm{rec}}$ ), which generates the perturbed phase shifts $\tilde{\delta}_{l}$ with the discrepancy $\epsilon=10^{-14}$. The corresponding numerical results are presented in Table 1 below. One can see that the first five phase shifts $\delta_{l}$ and $\tilde{\delta}_{l}$ of $q_{\text {orig }}$ and $q_{\text {rec }}$ correspondingly coincide, the remaining five phase shifts differ by the quantity of order $10^{-5}$, but the potentials $q_{\text {orig }}$ and $q_{\mathrm{rec}}$ are quite different.

\section{References}

[ I] B. Apagyi, G. Fndredi, P. Lévay, eds., Inverse and Algebraic Quantum Scattering theory, Proceedings, Lake Balaton, Hungary 1966, Lect. Notes in Phys. Vol. 488 (Springer, Berlin, 1997).

12] K. Chadan, P. Sabatier, Inverse Problems in Quantum Scattering Theory (Springer, Berlin, 1989).

[3] B.M. Levitan, Inverse Sturm-Liouville Problems (VNU, Utrecht, 1987).

[4] L. Landau, E. Lifshitz, Quantum Mechanics, Nonrelativistic Theory (Pergamon, Oxford, 1958).

15] R. Newton, Scattering Theory of Waves and Particles (Springer, Berlin, 1966).

16] A.G. Ramm, Multidimensional Inverse Scattering Problems (Longman/Wiley, New York, 1992), Russian translation of the expanded monograph (Mir, Moscow, 1994).

[7] A.G. Ramm, Inverse Problems 4 (1988) 877; 5 (1989) 255.

18] A.G. Ramm, Acta $\Lambda$ ppl. Math. 28 (1992) 1.

[9| A.G. Ramm, J.H. Arredondo, B.G. Izquierdo, J. Phys. A 31 (1998) L39.

[10] A.G. Ramm, Doklady Azerb. Acad. Sci. 22 (1966) 3.

111) A.G. Ramm, Inverse scattcring with part of the fixed-energy phase shifts, submitted (1998). 